\title{
A STRAIGHTFORWARD SYNTHESIS OF NOVEL 1,3,5-TRIAZINE-BASED MACROCYCLIC SCAFFOLDS
}

\author{
COSMIN V. CRIŞAN" ${ }^{a}$, NICULINA D. HĂDADEa, ION GROSU, \\ ANDREEA P. CRIŞAN ${ }^{a^{*}}$, ANAMARIA TEREC ${ }^{a^{*}}$
}

\begin{abstract}
The synthesis and structural investigation of a series of three macrocycles bearing 2,4,6-tris( $p$-phenylene)-1,3,5-triazine central units and oligoethyleneoxide bridges are reported. The approach implied the synthesis of non-symmetrical 1,3,5-triazines obtained via a cross-cyclotrimerization reaction, followed by a direct macrocyclization with the corresponding ditosylated oligoethyleneglycols. The obtained non-symmetrically C-substituted 1,3,5-triazines macrocycles were characterized by NMR spectroscopy and High-Resolution Mass Spectrometry (HRMS).
\end{abstract}

Keywords: macrocycles, 1,3,5-triazine, cross-cyclotrimerization, oligoethylene bridges

\section{INTRODUCTION}

The study of macrocyclic molecules has long been an underlying research area in supramolecular chemistry due to the interdisciplinary nature of the research work involved in investigating this type of molecules. Macrocycles are well known for their molecular recognition properties [1], but their applicability is far from being limited to this field. The versatility of macrocyclic molecules makes them suitable for a wider range of scientific interest and commercial applications such as materials science [2], catalysis [3], drug delivery systems [4]. Moreover, macrocyclic molecules have been employed as key components in the construction of non-symmetrical cryptands [5], a new class of cage molecules, which is beginning to sparkle an increased interest from the research community. As well, triazine-based macrocycles have been reported to be well suited as building blocks for covalent organic frameworks (COFs) [6].

a Babeş-Bolyai University, Faculty of Chemistry and Chemical Engineering, Department of Chemistry and SOOMCC, Cluj-Napoca, 11 Arany Janos, 400028, Cluj-Napoca, Romania *Corresponding authors: adiac@chem.ubbcluj.ro; asuciu@chem.ubbcluj.ro 
The 1,3,5-triazine motif is an intensely studied building block in supramolecular chemistry, due to its ease of synthesis and availability, being investigated on a large scale in various research areas such as $\mathrm{CO}_{2}$ capture/ storage and separation [7], materials science [8] and other industrial applications (e.g. furniture industry) [9]. Additionally, triazine-based host molecules proved to be highly efficient in capturing one of the most harmful class of pollutants, namely the polycyclic aromatic hydrocarbons (PAHs) [10].

In our previous work [11], we reported and investigated several cryptands exhibiting 1,3,5-triazine or 2,4,6-triphenyl-1,3,5-triazine aromatic units and oligoethyleneoxide bridges which displayed a high affinity for alkali metal ions (particularly for $\mathrm{Na}^{+}$and $\mathrm{K}^{+}$) and organic ammonium ions such as the dication of 1,5-naphthylenediamine.

Therefore, based on the versatility of the triazine scaffold and the prodigious applicability of macrocyclic molecules, we considered it of great interest to design and synthesize macrocycles exhibiting 1,3,5-triazine moieties and oligoethyleneglycol bridging units of various lengths, as presented in Figure1.

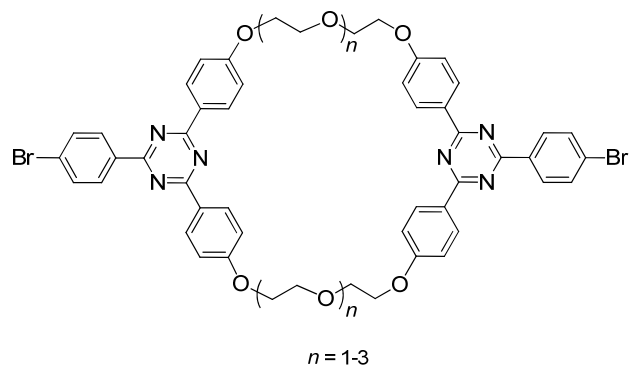

Figure 1. Target macrocycles with triazine units and oligoethyleneoxide bridges

\section{RESULTS AND DISCUSSIONS}

In order to access the target molecules, a two-step strategy was envisioned (Scheme 1). The key component in the construction of the macrocycles was the non-symmetrically C-2,4,6-trisubstituted 1,3,5-triazine unit 1a, which provides a precise control of the size, as well as the functionality of the final targets. Thus, the synthetic approach implied the preliminary preparation of the compound bearing suitable functional groups, followed, in the second step, by a direct macrocyclization reaction with selected ditosylated oligoethyleneglycols.

Symmetrically C-substituted 1,3,5-triazine derivatives are conventionally obtained by the cyclotrimerization of nitriles [12]. For our non-symmetrically C-2,4,6-trisubstituted 1,3,5-triazine unit, we had a different strategy, i.e., by introducing 4-bromobenzonitrile alongside 4-hydroxybenzonitrile (in 1:2.5 molar 
ratio) in a cross-cyclotrimerization process catalysed by triflic acid. As depicted in Scheme 1, a mixture of three differently C-substituted 1,3,5-triazines, 1a-c, was obtained, the desired 1a being predominant (values of partial conversions of starting nitriles based on the amount of material isolated by column chromatography are presented).

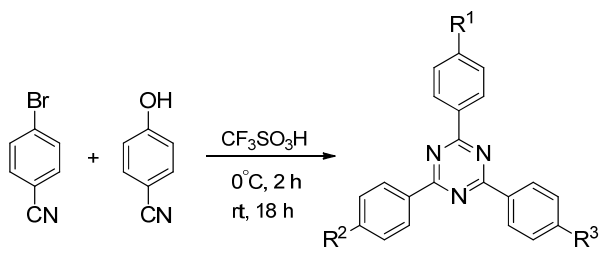

1a $\mathrm{R}^{1}=\mathrm{Br}, \mathrm{R}^{2}=\mathrm{R}^{3}=\mathrm{OH} \quad 49 \%$

1b $\mathrm{R}^{1}=\mathrm{OH}, \mathrm{R}^{2}=\mathrm{R}^{3}=\mathrm{Br} \quad 16 \%$

1c $\mathrm{R}^{1}=\mathrm{R}^{2}=\mathrm{R}^{3}=\mathrm{OH} \quad 23 \%$
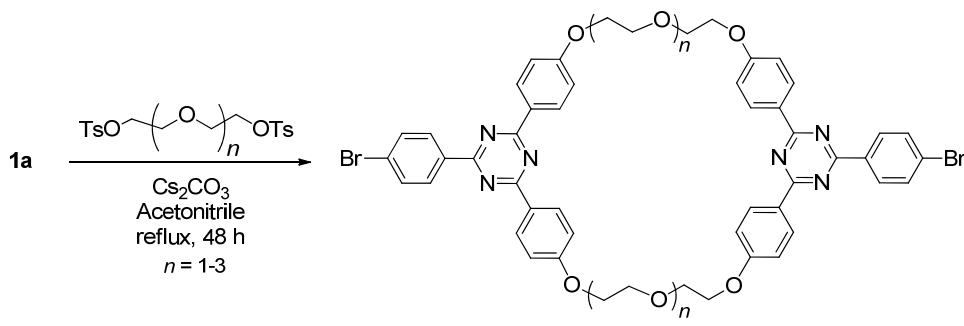

2, $n=1 \quad 18 \%$

$3, n=2 \quad 39 \%$

4, $n=3 \quad 29 \%$

Scheme 1. Synthesis of 1,3,5-triazine-based macrocycles

The desired non-symmetrically substituted 1,3,5-triazine 1a was used in the macrocyclization step. The target macrocycles 2-4 were thus obtained by directly reacting derivative $1 \mathrm{a}$ with readily available [13] ditosylated mono-, di- and triethyleneglycols using $\mathrm{Cs}_{2} \mathrm{CO}_{3}$ as a template and proton scavenger. In such doing, the triazine derivative $1 \mathrm{a}$ and the base, $\mathrm{Cs}_{2} \mathrm{CO}_{3}$, were dissolved in acetonitrile at high dilution and with slow addition of the solution containing the corresponding ditosylated glycol in order to favour the formation of the macrocycle over the oligomerization products. As shown in Scheme 1 and depending on the size $(n=1-3)$ of the oligoethyleneglycol, the above protocol afforded the desired macrocycles 2-4 with yields ranging from 18\% to $39 \%$. The highest yielding result was obtained when the ditosylated triethyleneglycol was used as bridging unit. 
Formation of macrocycles 2-4 was confirmed by NMR and HRMS experiments. The NMR spectra of all three macrocycles exhibited only a unique pattern of resonances, proving the symmetrical nature of the structures. Thus, the common ${ }^{1} \mathrm{H}$ NMR pattern for this series of compounds (Figure 2) consisted of two AA'-XX' coupling patterns in the aromatic region, with a very slight upfield shift for each of the compound's aromatic signals as the oligoethyleneglycol bridge grew longer. Also, the difference in the size of the compounds is clearly demonstrated by the appearance of an additional signal located in the 3.5-4.5 ppm range for each extra ethylene group in the chain.
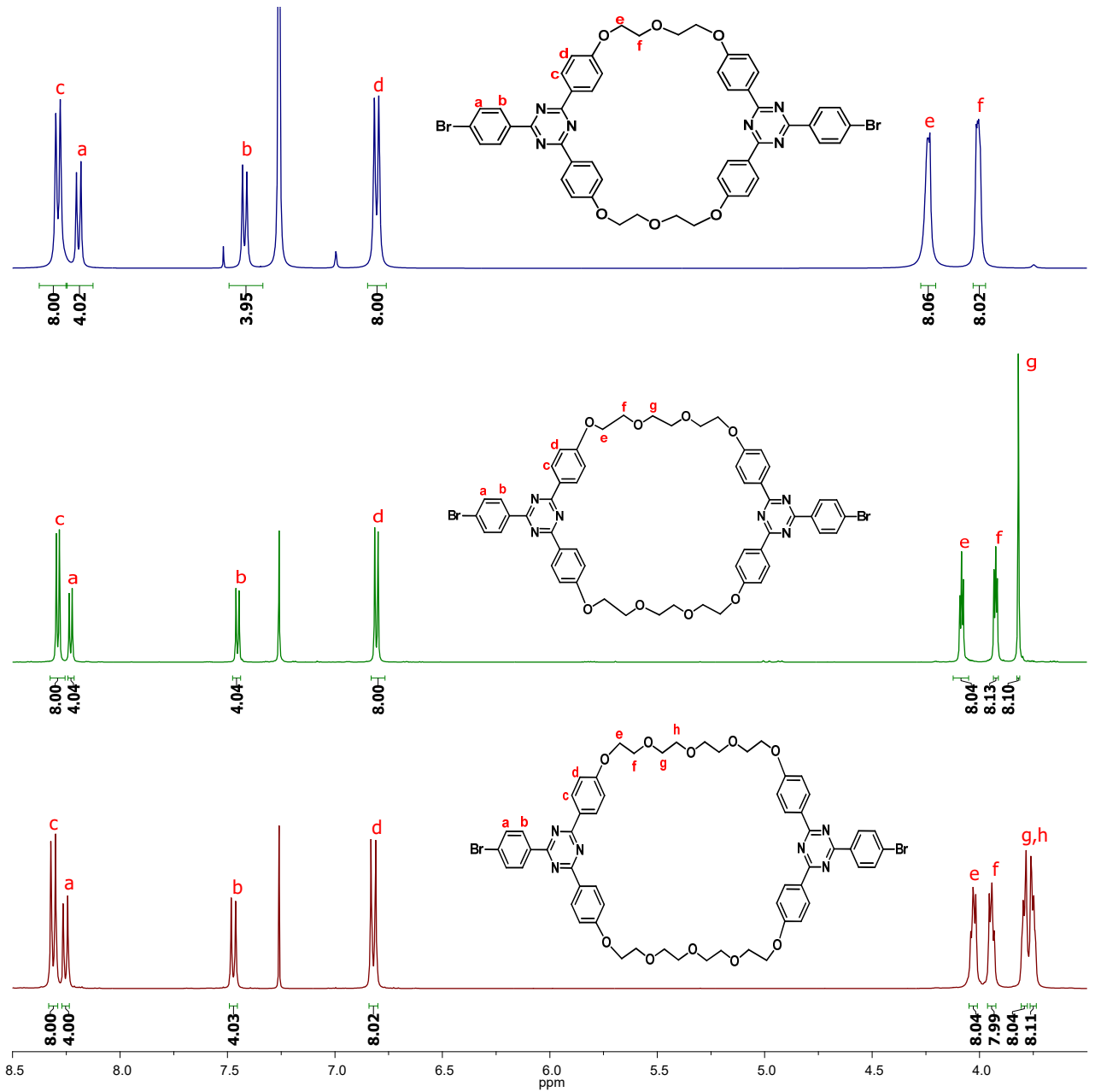

Figure 2. ${ }^{1} \mathrm{H} \mathrm{NMR}\left(600 \mathrm{MHz}, \mathrm{CDCl}_{3}\right)$ stacked spectra of macrocyclic derivatives 2-4 having different oligoethyleneglycol bridges 
Furthermore, the formation of the [2+2] product was confirmed by the HRMS spectra, which reveal in case of all three macrocycles the molecular peaks corresponding to the protonated species $[\mathrm{M}+\mathrm{H}]^{+}$at $\mathrm{m} / \mathrm{z}=981.1442$ for 2 , $\mathrm{m} / \mathrm{z}=1069.1969$ for 3 and $\mathrm{m} / \mathrm{z}=1557.2494$ for $\mathbf{4}$, respectively. Additionally, as presented in Figure 3, the expected molecular peaks exhibit a typical isotopic pattern of a di-brominated chemical species.
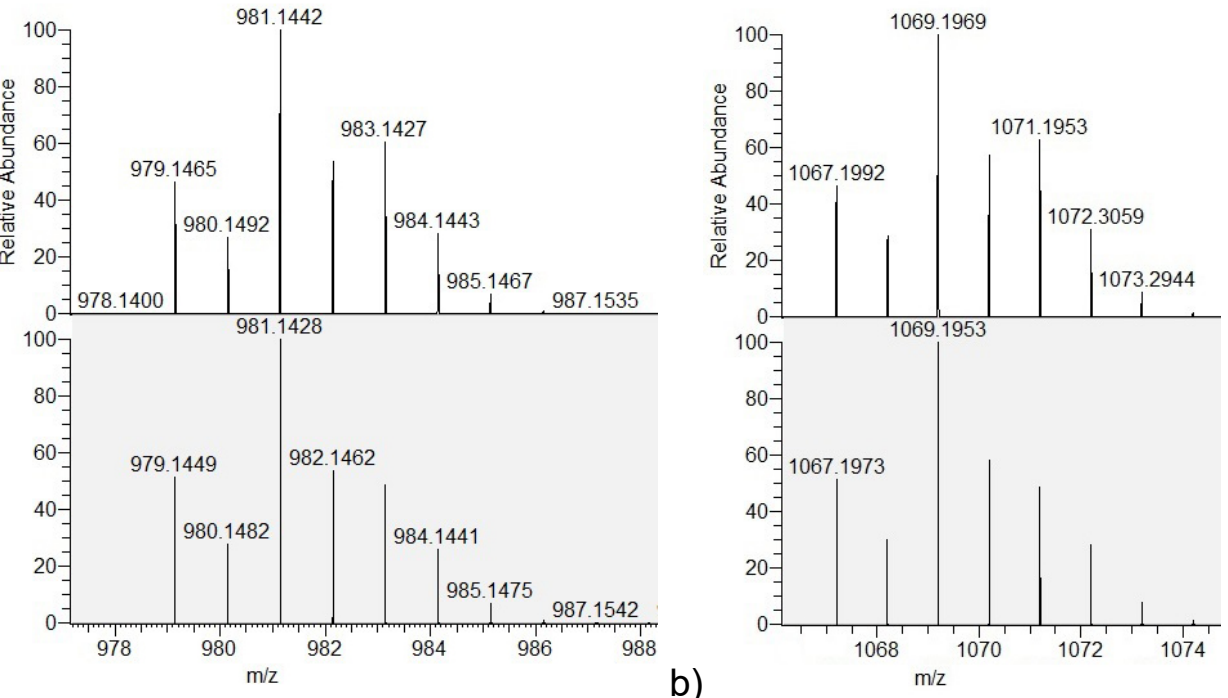

a)

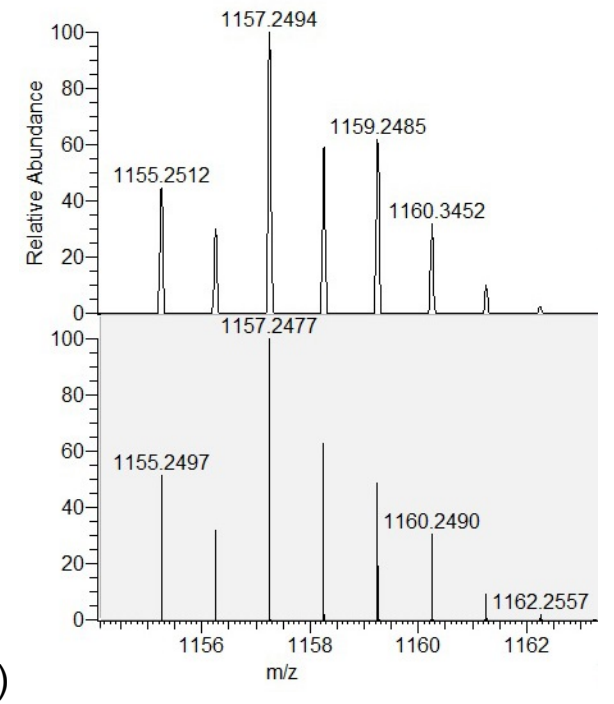

Figure 3. APCl(+)HRMS spectra of macrocycles 2 (a), 3 (b) and 4 (c). Comparison of the experimental spectra (top) and simulated isotopic patterns (bottom). 


\section{CONCLUSIONS}

In summary, a series of three novel 1,3,5-triazine-based macrocycles was synthesized and fully characterized. In order to access the target macrocycles, a novel non-symmetrical 2,4,6-C-trisubstituted-1,3,5-triazine derivative was obtained by adapting an efficient and innovative synthetic strategy. The straightforward procedure and the above findings prompt toward future design and synthesis of novel "intelligent organic molecules".

\section{EXPERIMENTAL SECTION}

General data: NMR spectra were recorded at room temperature, in $\mathrm{CDCl}_{3}$ or DMSO- $d_{6}$ as solvents, on instruments operating at 400 or $600 \mathrm{MHz}$ for ${ }^{1} \mathrm{H}$ and 100 or $150 \mathrm{MHz}$ for ${ }^{13} \mathrm{C}$ nuclei. Chemical shifts $(\delta)$ are reported in parts per million (ppm) using residual solvent peak as internal standard. High resolution mass spectra (HRMS) were recorded in positive mode, on a LTQ ORBITRAP XL spectrometer using external mass calibration. The ditosylated oligoethyleneglycols were obtained following a procedure described in the literature [13], while the other reagents were commercially available and were used without further purifications. Thin layer chromatography (TLC) was conducted on silica gel 60 F254 TLC plates. Solvents were dried and distilled under argon using standard procedures.

\section{Procedure for the unsymmetrical cyclotrimerization providing compounds 1a-c (Scheme 1)}

To ice-cooled $\left(0^{\circ} \mathrm{C}\right)$ trifluoromethanesulfonic acid $(0.300 \mathrm{~mL}, 0.510 \mathrm{~g}$, $3.30 \mathrm{mmol}$ ), an anhydrous $\mathrm{CHCl}_{3}$ solution $(20 \mathrm{~mL}$ ) containing 4-bromobenzonitrile $(0.100 \mathrm{~g}, 0.55 \mathrm{mmol})$ and 4-hydroxybenzonitrile $(0.164 \mathrm{~g}, 1.37 \mathrm{mmol})$ was added dropwise over 30 minutes. After additional stirring $(18 \mathrm{~h})$ at room temperature, water $(50 \mathrm{~mL})$ was added and the resulting precipitate was filtrated off, then washed with water to neutrality. The isolated dried solid was further separated by column chromatography on silica gel (eluent AcOEt/PE 1:1 v/v, Scheme 1) to provide compounds $1 \mathrm{a}(0.110 \mathrm{~g}, 49 \%$ partial conversion of 4-bromobenzonitrile), $1 \mathrm{~b}(0.042 \mathrm{~g}, 16 \%$ partial conversion of 4 -bromobenzonitrile $)$ and $1 \mathrm{c}(0.045 \mathrm{~g}, 23 \%$ partial conversion of 4-bromobenzonitrile).

2-(4-Bromophenyl)-4,6-bis(4-hydroxyphenyl)-1,3,5-triazine (1a). White solid; Mp.: $325^{\circ} \mathrm{C}$ with decomposition; $R_{\mathrm{f}}=0.65$ (AcOEt:PE 1:1 v/v). ${ }^{1} \mathrm{H}$ NMR $\left(400 \mathrm{MHz}, \mathrm{DMSO}-d_{6}\right) \delta_{\mathrm{H}}(\mathrm{ppm}): 10.36(\mathrm{~s}, 2 \mathrm{H}), 8.60\left(\mathrm{~d},{ }^{3} J_{\mathrm{H}, \mathrm{H}}=8.6 \mathrm{~Hz}, 2 \mathrm{H}\right)$, $8.56\left(\mathrm{~d},{ }^{3} \mathrm{~J}_{\mathrm{H}, \mathrm{H}}=8.8 \mathrm{~Hz}, 4 \mathrm{H}\right), 7.83\left(\mathrm{~d},{ }^{3} \mathrm{~J}_{\mathrm{H}, \mathrm{H}}=8.6 \mathrm{~Hz}, 2 \mathrm{H}\right), 6.98\left(\mathrm{~d},{ }^{3} \mathrm{~J}_{\mathrm{H} . \mathrm{H}}=8.8\right.$ $\mathrm{Hz}, 4 \mathrm{H}) \cdot{ }^{13} \mathrm{C}$ NMR $\left(100 \mathrm{MHz}, \mathrm{DMSO}-d_{6}\right) \delta_{\mathrm{c}}(\mathrm{ppm}): 170.5(\mathrm{C}=\mathrm{N}), 169.5(\mathrm{C}=\mathrm{N})$, 
162.1 (C=C, Ar), 135.1 (C=C, Ar), $131.9(\mathrm{HC}, \mathrm{Ar}), 130.8(\mathrm{HC}, \mathrm{Ar}), 130.4(\mathrm{HC}, \mathrm{Ar})$, $126.6(\mathrm{C}=\mathrm{C}, \mathrm{Ar}), 126.2(\mathrm{C}=\mathrm{C}, \mathrm{Ar}), 115.7(\mathrm{HC}, \mathrm{Ar})$. HRMS (APCl+): calculated for $\mathrm{C}_{21} \mathrm{H}_{15} \mathrm{BrN}_{3} \mathrm{O}_{2}[\mathrm{M}+\mathrm{H}]^{+}:$: 20.0342 ; found: 420.0332 .

2-(4-Hydroxyphenyl)-4,6-bis(4-bromophenyl)-1,3,5-triazine (1b). White solid; $R_{\mathrm{f}}=0.9\left(\right.$ AcOEt:PE 1:1 v/v). ${ }^{1} \mathrm{H}$ NMR $\left(400 \mathrm{MHz}\right.$, DMSO- $\left.d_{6}\right) \delta_{\mathrm{H}}$ (ppm): $10.44(\mathrm{~s}, 1 \mathrm{H}), 8.62\left(\mathrm{~d},{ }^{3} \mathrm{~J}_{\mathrm{H}, \mathrm{H}}=8.6 \mathrm{~Hz}, 4 \mathrm{H}\right), 8.58\left(\mathrm{~d},{ }^{3} \mathrm{~J}_{\mathrm{H}, \mathrm{H}}=8.8 \mathrm{~Hz}, 2 \mathrm{H}\right)$, $7.84\left(\mathrm{~d},{ }^{3} \mathrm{~J}_{\mathrm{H}, \mathrm{H}}=8.6 \mathrm{~Hz}, 4 \mathrm{H}\right), 7.00\left(\mathrm{~d},{ }^{3} \mathrm{~J}_{\mathrm{H} . \mathrm{H}}=8.8 \mathrm{~Hz}, 2 \mathrm{H}\right)$. HRMS (APCl+): calculated for $\mathrm{C}_{21} \mathrm{H}_{14} \mathrm{Br}_{2} \mathrm{~N}_{3} \mathrm{O}[\mathrm{M}+\mathrm{H}]^{+}$: 483.9478; found: 483.9465 .

2,4,6-Tris(4-hydroxyphenyl)-1,3,5-triazine (1c). White solid; $R_{\mathrm{f}}=0.4$ (AcOEt:PE 1:1 v/v). ${ }^{1} \mathrm{H}$ NMR $\left(600 \mathrm{MHz}\right.$, DMSO- $\left.d_{6}\right) \delta_{\mathrm{H}}(\mathrm{ppm}): 10.28(\mathrm{~s}, 3 \mathrm{H})$, $8.55\left(\mathrm{~d},{ }^{3} \mathrm{~J}_{\mathrm{H}, \mathrm{H}}=8.6 \mathrm{~Hz}, 6 \mathrm{H}\right), 6.97\left(\mathrm{~d},{ }^{3} \mathrm{~J}_{\mathrm{H}, \mathrm{H}}=8.6 \mathrm{~Hz}, 6 \mathrm{H}\right)$. HRMS (APCl+): calculated for $\mathrm{C}_{21} \mathrm{H}_{16} \mathrm{~N}_{3} \mathrm{O}_{3}[\mathrm{M}+\mathrm{H}]^{+}: 358.1186$; found: 358. 1178.

\section{Procedure for the synthesis of triazine-based macrocycles 2-4}

To a solution of $1 \mathrm{a}(0.100 \mathrm{~g}, 0.238 \mathrm{mmol})$ in $\mathrm{MeCN}(100 \mathrm{~mL}), \mathrm{Cs}_{2} \mathrm{CO}_{3}$ $(0.390 \mathrm{~g}, 1.19 \mathrm{mmol})$ was added and the resulting mixture was heated to $80^{\circ} \mathrm{C}$. The corresponding ditosylated oligoethyleneglycol $(0.238 \mathrm{mmol})$ solubilized in THF ( $5 \mathrm{~mL}$ ) was added dropwise over $6 \mathrm{~h}$ and the mixture was stirred for another $48 \mathrm{~h}$ until the reactants were fully consumed (TLC monitoring). After cooling, the solvent was removed under vacuum and the resulting mass was solubilized in $\mathrm{CHCl}_{3}(30 \mathrm{~mL})$ and washed with water $(40 \mathrm{~mL})$. The aqueous phase was further extracted with $\mathrm{CHCl}_{3}(3 \times 25 \mathrm{~mL})$, the combined organic layers were dried over $\mathrm{MgSO}_{4}$ and the solvent removed under reduced pressure. The isolated crude product was further separated by column chromatography on silica gel (gradient of eluent: Toluene/AcOEt 10:1 v/v for 2, Toluene/AcOEt 5:1 v/v for 3, Toluene/ AcOEt 3:1 v/v for 4$)$ to afford compounds $2(0.042 \mathrm{~g}, 18 \%$ partial conversion of $1 \mathrm{a}), 3(0.100 \mathrm{~g}, 39 \%$ partial conversion of $1 \mathrm{a}), 4(0.080 \mathrm{~g}, 29 \%$ partial conversion of 1a).

Macrocycle 2. White solid; $\mathrm{Mp} .: 193^{\circ} \mathrm{C} ; R_{\mathrm{f}}=0.3$ (Toluene:AcOEt 10:1 v/v). ${ }^{1} \mathrm{H}$ NMR $\left(400 \mathrm{MHz}, \mathrm{CDCl}_{3}\right) \delta_{\mathrm{H}}(\mathrm{ppm}): 8.29\left(\mathrm{~d},{ }^{3} J_{\mathrm{H}, \mathrm{H}}=8.7 \mathrm{~Hz}, 8 \mathrm{H}\right), 8.19$ $\left(\mathrm{d},{ }^{3} \mathrm{~J}_{\mathrm{H}, \mathrm{H}}=8.4 \mathrm{~Hz}, 4 \mathrm{H}\right), 7.42\left(\mathrm{~d},{ }^{3} \mathrm{~J}_{\mathrm{H}, \mathrm{H}}=8.4 \mathrm{~Hz}, 4 \mathrm{H}\right), 6.81\left(\mathrm{~d},{ }^{3} J_{\mathrm{H}, \mathrm{H}}=8.7 \mathrm{~Hz}, 8 \mathrm{H}\right)$, $4.24(\mathrm{~m}, 8 \mathrm{H}), 4.00(\mathrm{~m}, 8 \mathrm{H}) .{ }^{13} \mathrm{C} \mathrm{NMR}\left(150 \mathrm{MHz}, \mathrm{CDCl}_{3}\right) \delta_{\mathrm{c}}(\mathrm{ppm}): 170.3(\mathrm{C}=\mathrm{N}$, $\mathrm{Ar}), 169.7(\mathrm{C}=\mathrm{N}, \mathrm{Ar}), 161.9(\mathrm{C}=\mathrm{C}), 135.3(\mathrm{C}=\mathrm{C}), 131.6\left(\mathrm{HC}_{\mathrm{ar}}\right), 130.5\left(\mathrm{HC}_{\mathrm{ar}}\right)$, $130.0\left(\mathrm{HC}_{\mathrm{ar}}\right), 128.7(\mathrm{C}=\mathrm{C}), 127.0(\mathrm{C}=\mathrm{C}), 114.6\left(\mathrm{HC}_{\mathrm{ar}}\right), 69.9\left(\mathrm{CH}_{2}\right), 68.2\left(\mathrm{CH}_{2}\right)$. HRMS (APCl+): calculated for $\mathrm{C}_{50} \mathrm{H}_{41} \mathrm{Br}_{2} \mathrm{~N}_{6} \mathrm{O}_{6}[\mathrm{M}+\mathrm{H}]^{+}$: 981.1428; found: 981.1442 . 
Macrocycle 3. White solid; Mp.: $179{ }^{\circ} \mathrm{C} ; R_{\mathrm{f}}=0.23$ (Toluene:AcOEt $1: 1 \mathrm{v} / \mathrm{v}) .{ }^{1} \mathrm{H}$ NMR $\left(600 \mathrm{MHz}, \mathrm{CDCl}_{3}\right) \delta_{\mathrm{H}}(\mathrm{ppm}): 8.29\left(\mathrm{~d},{ }^{3} \mathrm{~J}_{\mathrm{H}, \mathrm{H}}=8.7 \mathrm{~Hz}, 8 \mathrm{H}\right)$, $8.23\left(\mathrm{~d},{ }^{3} \mathrm{~J}_{\mathrm{H}, \mathrm{H}}=8.4 \mathrm{~Hz}, 4 \mathrm{H}\right), 7.45\left(\mathrm{~d},{ }^{3} \mathrm{~J}_{\mathrm{H}, \mathrm{H}}=8.4 \mathrm{~Hz}, 4 \mathrm{H}\right), 6.81\left(\mathrm{~d},{ }^{3} \mathrm{~J}_{\mathrm{H}, \mathrm{H}}=8.7 \mathrm{~Hz}\right.$, $8 \mathrm{H}), 4.09(\mathrm{~m}, 8 \mathrm{H}), 3.92(\mathrm{~m}, 8 \mathrm{H}), 3.82(\mathrm{~s}, 8 \mathrm{H}) .{ }^{13} \mathrm{C} \mathrm{NMR}\left(150 \mathrm{MHz}, \mathrm{CDCl}_{3}\right) \delta_{\mathrm{C}}$ (ppm): $170.4(\mathrm{C}=\mathrm{N}), 169.7(\mathrm{C}=\mathrm{N}), 162.4(\mathrm{C}=\mathrm{C}, \mathrm{Ar}), 135.3(\mathrm{C}=\mathrm{C}, \mathrm{Ar}), 131.6$ ( $\mathrm{HC}, \mathrm{Ar}), 130.6$ (HC, Ar), $130.2(\mathrm{HC}, \mathrm{Ar}), 128.6(\mathrm{C}=\mathrm{C}, \mathrm{Ar}), 127.0(\mathrm{C}=\mathrm{C}, \mathrm{Ar})$, 114.2 ( $\mathrm{HC}, \mathrm{Ar}), 71.2\left(\mathrm{CH}_{2}\right), 69.8\left(\mathrm{CH}_{2}\right), 67.4\left(\mathrm{CH}_{2}\right)$. HRMS (APCl+): calculated for $\mathrm{C}_{54} \mathrm{H}_{49} \mathrm{Br}_{2} \mathrm{~N}_{6} \mathrm{O}_{8}[\mathrm{M}+\mathrm{H}]^{+}:$1069.1953; found: 1069.1969.

Macrocycle 4. White solid; Mp.: $163^{\circ} \mathrm{C} ; R_{\mathrm{f}}=0.15$ (Toluene:AcOEt 3:1 $\mathrm{v} / \mathrm{v}) .{ }^{1} \mathrm{H}$ NMR $\left(400 \mathrm{MHz}, \mathrm{CDCl}_{3}\right) \delta_{\mathrm{H}}(\mathrm{ppm}): 8.31\left(\mathrm{~d},{ }^{3} \mathrm{~J}_{\mathrm{H}, \mathrm{H}}=8.8 \mathrm{~Hz}, 8 \mathrm{H}\right), 8.25(\mathrm{~d}$, $\left.{ }^{3} J_{\mathrm{H}, \mathrm{H}}=8.5 \mathrm{~Hz}, 4 \mathrm{H}\right), 7.47\left(\mathrm{~d},{ }^{3} \mathrm{~J}_{\mathrm{H}, \mathrm{H}}=8.5 \mathrm{~Hz}, 4 \mathrm{H}\right), 6.82\left(\mathrm{~d},{ }^{3} \mathrm{~J}_{\mathrm{H}, \mathrm{H}}=8.8 \mathrm{~Hz}, 8 \mathrm{H}\right)$, $4.03(\mathrm{~m}, 8 \mathrm{H}), 3.95(\mathrm{~m}, 8 \mathrm{H}), 3.79(\mathrm{~m}, 8 \mathrm{H}), 3.75(\mathrm{~m}, 8 \mathrm{H}) .{ }^{13} \mathrm{C} \mathrm{NMR}(150 \mathrm{MHz}$, $\left.\mathrm{CDCl}_{3}\right) \delta_{\mathrm{C}}(\mathrm{ppm}): 170.4(\mathrm{C}=\mathrm{N}), 169.7(\mathrm{C}=\mathrm{N}), 162.5(\mathrm{C}=\mathrm{C}, \mathrm{Ar}), 135.3(\mathrm{C}=\mathrm{C}, \mathrm{Ar})$, 131.6 (HC, Ar), $130.7(\mathrm{HC}, \mathrm{Ar}), 130.3(\mathrm{HC}, \mathrm{Ar}), 128.6(\mathrm{C}=\mathrm{C}, \mathrm{Ar}), 127.1(\mathrm{C}=\mathrm{C}$, Ar), $114.2(\mathrm{HC}, \mathrm{Ar}), 71.3\left(\mathrm{CH}_{2}\right), 70.8\left(\mathrm{CH}_{2}\right), 69.9\left(\mathrm{CH}_{2}\right), 67.7\left(\mathrm{CH}_{2}\right)$. HRMS (APCl+): calculated for $\mathrm{C}_{58} \mathrm{H}_{57} \mathrm{Br}_{2} \mathrm{~N}_{6} \mathrm{O}_{10}[\mathrm{M}+\mathrm{H}]^{+}:$1157.2477; found: 1157.2494.

\section{ACKNOWLEDGMENTS}

Financial support provided by Romanian National Authority for Scientific Research and Innovation, CNCS-UEFISCDI, Project number PN-II-ID-PCE2012-4-0248, is gratefully acknowledged.

\section{REFERENCES}

1. (a) Z. Liu; S.K.M. Nalluri; J.F. Stoddart; Chem. Soc. Rev., 2017, 46, 2459-2478; (b) I. Medruţ; R. Turdean; R. Gropeanu; F. Pop; L. Toupet; N.D. Hădade; E. Bogdan; I. Grosu; Tetrahedron Lett., 2013, 54, 1107-1111.

2. D. Shetty; J.K. Khedkar; K.M. Parkad; K. Kim; Chem. Soc. Rev., 2015, 44, 8747-8761.

3. Y. Liu; Y. Chen; H.-Y. Zhang; Handbook of Macrocyclic Supramolecular Assembly, Springer Nature Singapore Pte Ltd., 2019.

4. X. Ma; Y. Zhao; Chem. Rev., 2015, 115, 7794-7839.

5. (a) M. Zhang; X. Yan; F. Huang; Z. Niu; H.W. Gibson; Chem. Commun., 2020, 56, 766-769; (b) M. Otte; M. Lutz; R.J.M. Klein Gebbink; Eur. J. Org. Chem., 2017, 12, 1657-1661; (c) X. Ji; M. Zhang; X. Yan; J. Li F. Huang; Chem. Commun., 2013, 49, 1178-1180; (d) P. Wei; H. Wang; K. Jie; F. Huang; Chem. Commun., 2017, 53, 1688-1691. 
6. (a) G. Wang; T. Zhoua; Y. Lei; RSC Adv., 2020, 10, 11557-11564; (b) P. Bhanja; K. Bhunia; S.K. Das; D. Pradhan; Ry. Kimura; Y. Hijikata; S. Irle; A. Bhaumik; Chem. Sus. Chem., 2017, 10, 921-929.

7. P. Puthiaraj; Y.-R. Lee; S. Zhanga; W.-S. Ahn; J. Mater. Chem. A., 2016, 4, 16288-16311.

8. (a) G.J.A.A. Soler-Illia; O. Azzaroni; Chem. Soc. Rev., 2011, 40, 1107-1150; (b) M.A. Mintzer; M.W. Grinstaff; Chem. Soc. Rev., 2011, 40, 173-190; (c) Y. Gao; Y. Qu; T. Jiang; H. Zhang; N. He; B. Li; J. Wu; J.J. Hua; Mat. Chem. C, 2014, 2, 63536358; (d) A. Modak; M Pramanik; S.Inagaki; A. Bhaumik; J. Mat. Chem. A, 2014, 2, 11642-11650; (e) P. Puthiaraj; K. Pitchumani; Chem.-Eur. J., 2014, 20, 87618770; (f) J. Lim; M. A. Mintzer; L.M. Perez; E.E. Simanek; Org. Lett., 2010, 12, 1148-1151; (g) V. Percec; M.R. Imam; M. Peterca; W.D. Cho; P.A. Heiney; Isr. J. Chem., 2009, 49, 55-70.

9. (a) C.B. Verma; M.A. Quraishi; E.E. Ebenso; Inter. J. Electrochem. Sci., 2014, 9, 5537; (b) L.-J. Shao; G.-Y. Xing; C.-Z. Qi; Chem. Pap., 2014, 68, 983; (c) M. N.S. Rad; S. Behrouz; A. Movahedian; M.M. Doroodmand; Y. Ghasemi; S. Rasoul-Amini; A.-R.A. Gandomani; R. Rezaie; Helv. Chim. Acta, 2013, 96, 688.

10. (a) R.- F. Zhang; W.- J. Hu; Y.A. Liu; X.- L. Zhao; J.- S. Li; B. Jiang; K. Wen; J. Org. Chem., 2016, 81, 5649-5654; (b) J. Samanta; R. Natrajan; Org. Lett., 2016, 18, 3394-3397.

11. (a) C.V. Crișan; A. Terec; N.D. Hădade; I. Grosu; Tetrahedron, 2015, 71, 6888-6893; (b) F. Pop; C. Socaci; A. Terec; E. Condamine; R.A. Varga; C.I. Raţ; J. Roncali; I. Grosu; Tetrahedron, 2012, 41, 8581-8588; (c) A. Woiczechowski-Pop; D. Gligor; A. Bende; C. Varodi; E. Bogdan; A. Terec; I. Grosu; Supramol. Chem., 2015, 27, 52-58; (d) C. Lar; A. Woiczechowski-Pop; A. Bende; I.G. Grosu; N. Miklasova; E. Bogdan; N.D. Hădade; A. Terec; I Grosu; Beilstein J. Org. Chem., 2018, 14, 1370-1377.

12. a) B.P. Dash; R. Satapathy; J.A. Maguire; N.S. Hosmane; Organometallics, 2010, 29, 5230-5235; b) A. Woiczechowski-Pop; I.L. Dobra; G.D. Roiban; A. Terec; I. Grosu; Synth. Commun., 2012, 42, 3579-3588; c) F. Piron; C. Oprea; C. Cismaş; A Terec; J. Roncali; I. Grosu; Synthesis, 2010, 1639-1644.

13. K.M. Bonger; R.J.B.H.N. van den Berg; LH. Heitman; A.P.I. Jzerman; J. Oosterom; C.M. Timmers; H.S. Overkleefta; G.A. van der Marel; Bioorg. Med. Chem., 2007, 15, 4841-4856. 